\section{Quality assessment in gastroenterological research: publication rates of meta-analyses}

\section{Daryl Ramai ${ }^{a}$, Mohamed Barakat ${ }^{\mathrm{b}}$, Andrew Ofosu', Amaninder Dhaliwalc, Douglas G. Adler ${ }^{\mathrm{d}}$}

The Brooklyn Hospital Center, NY; University of Nebraska Medical Center, NE; University of Utah School of Medicine, Huntsman Cancer Center, Salt Lake City, UT, USA

Meta-analysis is a formal quantitative study design used to systematically assess and pool the results of smaller research studies [1]. Meta-analyses allow researchers to derive more concrete conclusions in the absence of larger clinical studies. Smaller clinical studies may contain limited or conflicting results, which can complicate clinical decision-making based on their conclusions. As a result, meta-analysis plays a central role in evidence-based medicine by integrating multiple studies and overcoming external validity limitations $[2,3]$.

A review of the meta-analyses presented at large gastroenterology meetings suggests that their number is increasing. We hypothesize that, while the number of metaanalyses is high, only a minority are published as full text. We aimed to compare the publication rates of meta-analysis abstracts presented at Digestive Disease Week (DDW) from 2014-2017.

We identified abstracts of meta-analyses presented at DDW from 2014-2017 published in supplements of the American Gastroenterological Association's (AGA) journal Gastroenterology and the American Society for Gastrointestinal Endoscopy's (ASGE) journal Gastrointestinal Endoscopy. Abstracts were further assessed by cross-referencing the PubMed database and Google Scholar to determine fulltext manuscript publication status. Information on country of study, number of authors, male or female lead or senior authorship, university or community program, number of studies, and sample size was extracted. Descriptive analysis of abstract and full-length paper publication characteristics was performed using SPSS v25 IBM (USA).

During the study period, 523 meta-analyses were presented at DDW (330 AGA abstracts and 193 ASGE abstracts). Of those, 96 AGA and 44 ASGE abstracts were published as fulllength manuscripts. The US contributed 374 (73.9\%) metaanalyses, compared to 53 (10.5\%) from Europe, 55 (10.9\%) from Asia, and 24 (4.7\%) from Canada. Ninety-five percent of abstracts were from university programs, compared to $5 \%$ from community programs $(\mathrm{P}<0.001)$. Male lead and senior authors accounted for $74 \%$ and $84 \%$ of authors, respectively. During the study period, only $26.6 \%$ of abstracts were eventually published as full-length manuscripts (523 abstracts vs. 139 manuscripts, $\mathrm{P}<0.001$ ). Approximately $41 \%$ of abstracts in 2014 , $6 \%$ of abstracts in 2015, 24\% of abstracts in 2016 and $37 \%$ of abstracts in 2017 were published as full-length papers (Fig. 1). Abstracts and full-text manuscripts had a similar median number of studies included for analysis ( 11 vs. $12, \mathrm{P}>0.05$, respectively).

Our report demonstrated that while the number of meta-analyses presented at DDW is growing, less than 30\% of abstracts are translated into full-length manuscripts. Furthermore, the numbers of studies that met the inclusion criteria and were analyzed in either abstract or manuscript format were similar. This raises the question: "of what quality are these meta-analyses?"

Lui et al (2017) assessed the quality of 127 gastroenterologybased full-length manuscripts and reported that the methodological reporting quality of meta-analyses has improved with compliances to the PRISMA statement and AMSTAR checklist [4]. However, very little information is known about the quality of systematic reviews and metaanalyses submitted to conferences.

With the advent of new and user-friendly software, pooled analyses are relatively easier to perform. What is more, these analyses can be performed by operators with little to no statistical training. Anecdotally, some meta-analyses are simply a repeat of studies that have been previously performed with the addition of 1-2 recent original research studies, while other studies are underpowered, which brings into question the need for performing a meta-analysis.

While abstracts do play a role in distilling information for the reader, full-length manuscripts are essential in helping readers evaluate the integrity and quality of research. Hence, without
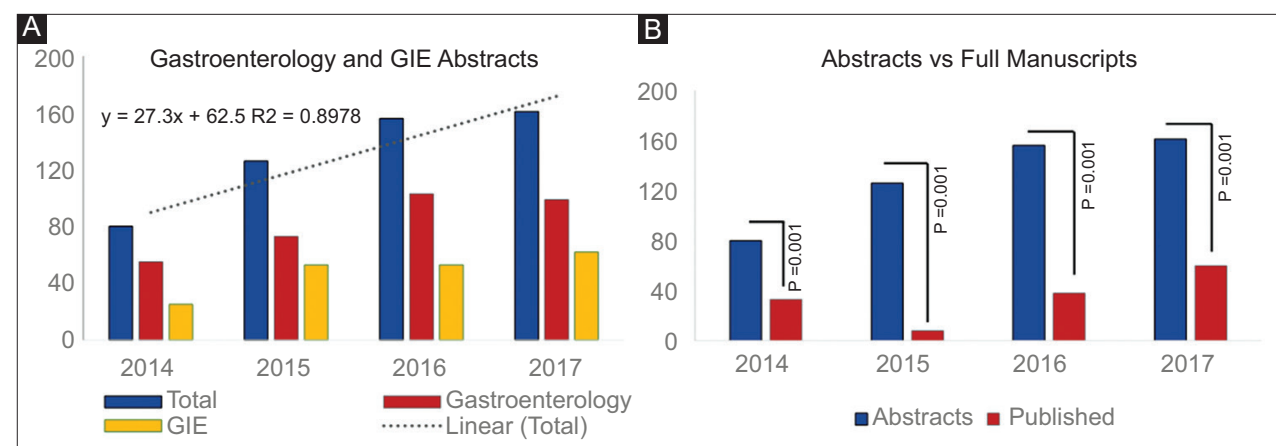

Figure 1 (A) Gastroenterology and Gastrointestinal Endoscopy (GIE) abstracts presented from 2014-2017. (B) Comparison of abstracts and fulllength manuscript publications 
an assessment of research methodology, conclusions drawn from meta-analyses abstracts are limited, at best. Additionally, authors and reviewers should evaluate the significance and limitations of systematic reviews and meta-analyses. Despite presenting the best available evidence, a meta-analysis based on low-quality studies might be misleading and must be interpreted with caution [5]. To this end, assessing the quality of studies is also limited within an abstract format; thus, a fulllength manuscript is needed.

Conference abstracts are not a substitute for full-length manuscripts. A consensus statement from the PRISMA for Abstracts Group noted that abstracts should be robust enough in presenting a clear and truthful account of the intended research [6]. More attention should be paid by authors and society abstract reviewers to the quality of systematic reviews and meta-analyses, given their central role in providing robust evidence-based medicine in the field of gastroenterology.

\section{References}

1. Gurevitch J, Koricheva J, Nakagawa S, Stewart G. Meta-analysis and the science of research synthesis. Nature 2018;555:175-182.

2. Fried M, Quigley EM, Hunt RH, et al. Is an evidence-based approach to creating guidelines always the right one? Nat Clin Pract Gastroenterol Hepatol 2008;5:60-61.
3. Kanwal F, White D. "Systematic reviews and meta-analyses" in clinical gastroenterology and hepatology. Clin Gastroenterol Hepatol 2012;10:1184-1186.

4. Liu P, Qiu Y, Qian Y, et al. Quality of meta-analyses in major leading gastroenterology and hepatology journals: a systematic review. J Gastroenterol Hepatol 2017;32:39-44.

5. Yuan Y, Hunt RH. Systematic reviews: the good, the bad, and the ugly. Am J Gastroenterol 2009;104:1086-1092.

6. Beller EM, Glasziou PP, Altman DG, et al; PRISMA for Abstracts Group. PRISMA for abstracts: reporting systematic reviews in journal and conference abstracts. PLoS Med 2013;10:e1001419.

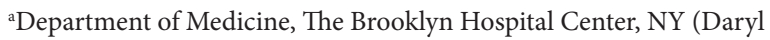
Ramai); ${ }^{b}$ Division of Gastroenterology and Hepatology, The Brooklyn Hospital Center, NY (Mohamed Barakat, Andrew Ofosu); 'Division of Gastroenterology, University of Nebraska Medical Center, NE

(Amaninder Dhaliwal); ${ }^{\mathrm{d} D i v i s i o n}$ of Gastroenterology and Hepatology, University of Utah School of Medicine, Huntsman Cancer Center, Salt Lake City, UT (Douglas G. Adler), USA

Conflict of Interest: None

Correspondence to: Douglas G. Adler MD, FACG, AGAF, FASGE, Professor of Medicine, Director of Therapeutic Endoscopy, Director, GI Fellowship Program, Gastroenterology and Hepatology, University of Utah School of Medicine, 30N 1900E 4R118, Salt Lake City, Utah 84132, USA, e-mail: Douglas.adler@hsc.utah.edu

Received 10 April 2020; accepted 22 April 2020; published online 15 May 2020

DOI: https://doi.org/10.20524/aog.2020.0493 